\title{
Konjunkturschlaglicht
}

\section{Rohstoffpreisentwicklung im Jahr 2019}

Die Entwicklungen auf den Rohstoffmärkten waren 2019 insbesondere durch globale Konflikte geprägt: einerseits durch den Handelskrieg zwischen den USA und China, der bereits seit Mitte 2018 anhält, und vor allem die Preise für Industriemetalle, aber auch die für Agrarrohstoffe im Jahr 2019 deutlich beeinflusste; andererseits durch die zunehmenden Spannungen zwischen den USA und dem Iran, die die Preise auf den Rohölmärkten antrieben. Trotz zeitweiser angespannter weltpolitischer Situation lag der durchschnittliche Rohölpreis 2019 unter dem Niveau des Jahres 2018.

Der HWWI-Rohstoffpreisindex ${ }^{1}$ ist 2019 durchschnittlich um $12 \%$ im Vergleich zum Vorjahr gesunken und auch die Teilindizes für Energierohstoffe, Industrierohstoffe und Nahrungs- und Genussmittel sind gefallen. Während die Preise für Energierohstoffe im Jahresdurchschnitt um $14 \%$ und die für Nahrungs- und Genussmittel um $5 \%$ sanken, blieben die Preise für Industrierohstoffe nahezu unverändert und wiesen lediglich eine negative Wachstumsrate von $0,5 \%$ auf (vgl. Abbildung 1).

Auch die Preise für Rohöl sind 2019 im Vergleich zu 2018 durchschnittlich gesunken. Dabei lag der Durchschnitts-

(C) Der/die Autor(en) 2020. Open Access: Dieser Artikel wird unter der Creative Commons Namensnennung 4.0 International Lizenz (https:// creativecommons.org/licenses/by/4.0/deed.de) veröffentlicht.

Open Access wird durch die ZBW - Leibniz-Informationszentrum Wirtschaft gefördert.

1 Der HWWI-Rohstoffpreisindex bildet die preislichen Veränderungen der 31 wichtigsten internationalen Rohstoffe aus den Bereichen Energie, Nahrungs- und Genussmittel und Industrierohstoffe ab.

\section{Abbildung 1}

\section{HWWI-Rohstoffpreisindex}



Quelle: Hamburgisches WeltwirtschaftsInstitut (HWWI). preis für ein Barrel der europäischen Referenzsorte Brent 2019 bei 64 US- $\$$ und für die amerikanische Referenzsorte West Texas Intermediate (WTI) bei 57 US-\$. Bei Betrachtung der Rohölpreisentwicklung (vgl. Abbildung 2) werden vier unterschiedliche Phasen deutlich. In der ersten Phase, die das 4. Quartal 2018 umfasst, fielen die Rohölpreise kontinuierlich und spiegelten die Ausweitung des Ölangebots aufgrund der stetig ansteigenden Schieferölproduktion der USA wider. Mitte 2018 überholten die USA SaudiArabien und Russland und entwickelten sich zum weltweit größten Ölproduzenten. Zusätzlich drückte das verlangsamte globale Wirtschaftswachstum, ausgelöst unter anderem durch den Handelskrieg zwischen China und den USA, die weltweite Nachfrage nach Rohöl.

Um dem Preisverfall entgegenzuwirken, einigte sich die OPEC $+{ }^{2}$ im Dezember 2018 auf eine Kürzung der Fördermenge von 1,2 Mio. Barrel Rohöl pro Tag. ${ }^{3}$ Die Angebotsverknappung erzielte die gewünschte Wirkung und die Preise stiegen bis Ende April 2019 auf ein Jahresmaximum von 75 US-\$ pro Barrel für Brent und 66 US-\$ pro Barrel für WTI-Rohöl (vgl. Phase 1, Abbildung 2). Die Rohölpreise wurden zusätzlich durch die von den USA verhängten Sanktionen gegen Venezuela und Iran getrieben. Vene-

2 Die Organisation der erdölexportierenden Ländern (OPEC) umfasst zurzeit die folgenden Mitglieder: Algerien, Angola, Ecuador, Äquatorialguinea, Gabun, Iran, Irak, die Republik Kongo, Kuwait, Libyen, Nigeria, Saudi-Arabien, die Vereinigten Arabischen Emirate und Venezuela. OPEC+ umschließt noch weitere Förderländer inklusive Russland.

3 OPEC: 175th Meeting, Wien, 6.12.2018, https://www.opec.org/opec_ web/en/press_room/5278.htm (3.2.2020).

Abbildung 2

Entwicklung der Rohölpreise

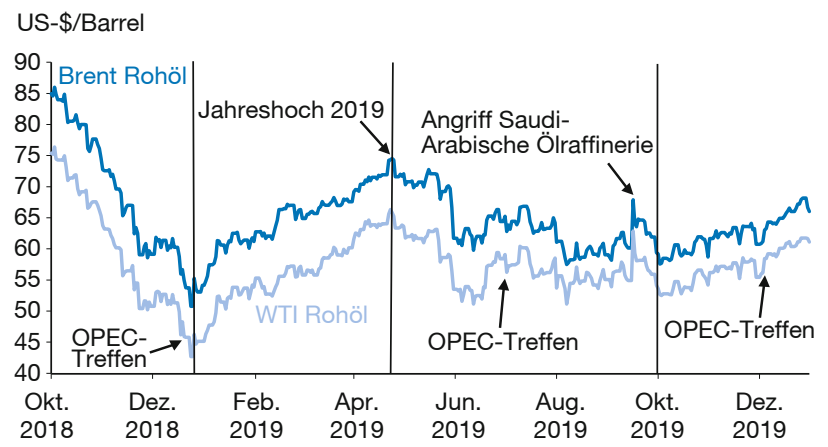

Quellen: Macrobond; U.S. Energy Information Administration. 
zuela besitzt weltweit die größten Ölreserven. Aufgrund der Wirtschaftskrise und der Sanktionen der USA ist die venezolanische Ölförderung in den letzten Jahren stark eingebrochen und die Ölexporte sind deutlich gesunken. ${ }^{4}$ 2019 haben die USA das Nuklearabkommen mit dem Iran einseitig aufgekündigt und Sanktionen auf iranische Rohölexporte verhängt. Seither ist die Rohölförderung des Irans ebenfalls rückläufig.

Obwohl sich in Phase 3 ab Mai 2019 der Konflikt zwischen den USA und Iran erneut verschärfte, setzte sich der Aufwärtstrend auf den Märkten für Rohöl nicht weiter fort. Die USA verstärkten die wirtschaftlichen Sanktionen gegen den Iran, der Iran wiederum drohte mit einer Blockade der StraBe von Hormus ${ }^{5}$ für internationale Rohöltransporte. Erneut zeigte sich, dass die Rohölmärkte gut versorgt sind, da diese Ereignisse nur zu kurzfristigen Anstiegen der Ölpreise führten. Daran änderte auch nichts, dass die OPEC+ Anfang Juli entschied, die Drosselung der eigenen Ölförderung für weitere neun Monate beizubehalten. ${ }^{6}$ Am 16.9.2019 stiegen die Preise enorm, als nach einem Drohnenangriff erhebliche Teile der weltweit größten saudi-arabischen Rohölraffinerie beschädigt wurden. Da Saudi-Arabien in der Lage war, die Produktionskapazitäten innerhalb kürzester Zeit wiederaufzubauen, stiegen die Preise nur kurzzeitig an und fielen anschließend auf ein geringeres Niveau als noch vor dem Angriff. Das Jahr 2019 endete mit einem leichten Aufwärtstrend auf den Rohölmärkten. Im Dezember 2019 einigte sich die OPEC+ erneut auf eine Kürzung der Fördermenge um zusätzliche 500000 Barrel Öl pro Tag (vgl. Phase 4, Abbildung 2). ${ }^{7}$

Der Handelskonflikt zwischen den USA und China beeinflusste 2019 auch die Entwicklung des Index für Industrierohstoffe $^{8}$ (vgl. Abbildung 1). Die bilateral verhängten Strafzölle schwächten das chinesische Wirtschaftswachstum und reduzierten insbesondere die Nachfrage nach Industriemetallen, was zu Preisreduktionen auf den Märkten für Stahlschrott und NE-Metalle führte. ${ }^{9}$ Dass der Index

4 Vgl. U.S. Energy Information Administration: Country Analysis Executive Summary: Venezuela, 7.1.2019, https://www.eia.gov/international/content/analysis/countries_long/Venezuela/venezuela_exe.pdf (3.2.2020).

5 Die Straße von Hormus ist für den internationalen Rohölhandel von großer Bedeutung, da ein Drittel der internationalen Öltransporte auf diesem Seeweg verschifft wird. Vgl. U.S. Energy Information Administration: The Strait of Hormuz is the world's most important oil transit chokepoint, 20.6.2019, https://www.eia.gov/todayinenergy/detail.php?id=39932 (11.2.2020).

6 OPEC: 176th Meeting, Wien, 1.7.2019, https://www.opec.org/opec_ web/en/press_room/5591.htm (3.2.2020).

7 OPEC: 177th Meeting, Wien, 5.12.2019, https://www.opec.org/opec_ web/en/press_room/5794.htm (3.2.2020).

8 Der Index für Industrierohstoffe umfasst die Preise der wichtigsten agrarischen Industrierohstoffe, die Preise für NE-Metalle (Aluminium, Blei, Kupfer, Nickel, Zink, Zinn) sowie die für Eisenerz und Stahlschrott.

9 Im Jahr 2019 erreichte das Wachstum der chinesischen Industrieproduktion einen mehrjährigen Tiefststand. Vgl. Weltbank: Global Outlook 2020, https://openknowledge.worldbank.org/bitstream/ handle/10986/33044/211469-Ch01.pdf (3.2.2020). für Industrierohstoffe sich im Jahresdurchschnitt dennoch nahezu unverändert zeigte, ist auf die starken Preisanstiege auf den Märkten für Eisenerz in der ersten Jahreshälfte 2019 zurückzuführen. Seit Januar 2019 stiegen die Preise für Eisenerz deutlich an, da bei einem Dammbruch in der brasilianischen Stadt Brumadinho große Teile einer wichtigen Eisenerzmine zerstört wurden und sich dadurch das weltweite Angebot an Eisenerz stark verknappte.

Auch der Index für Nahrungs- und Genussmittel fiel 2019 verglichen mit dem Vorjahr. Der Handelskonflikt beeinflusste ebenfalls die Preise für Agrarrohstoffe, hier insbesondere für Sojabohnen und Getreide. Die rückläufigen chinesischen Importe von US-amerikanischen Agrarprodukten reduzierten die Nachfrage und drückten die Preise. Die Kaffeepreise wurden zwar nicht durch die Spannungen zwischen China und den USA beeinflusst, sanken 2019 aber ebenfalls durchschnittlich. Der seit 2016 fallende Preistrend auf den Kaffeemärkten ist auf die Überkapazitäten, die sich trotz stetig ansteigender Nachfrage halten, zurückzuführen. ${ }^{10}$ Ende 2019 waren sowohl auf den Märkten für Industrierohstoffe als auch auf den Märkten für Agrarrohstoffe Preisanstiege zu beobachten, da China und die USA sich auf ein Teilabkommen einigten. Das Teilabkommen entschärfte die Spannungen zwischen den beiden Weltmächten und erhöhte die Erwartungen auf eine Verbesserung der globalen Wirtschaftslage.

Globale Verwerfungen beeinflussten 2019 die Preise auf den internationalen Rohstoffmärkten und erhöhten die Unsicherheit. Die Spannungen zwischen dem Iran und den USA verschärften sich zunehmend. Die Rohölmärkte reagierten nur kurzfristig mit Preiserhöhungen, was darauf hinweist, dass die Märkte gut versorgt sind. Das ansteigende Schieferölangebot aus den USA und die fallende Nachfrage aufgrund des rückläufigen Wirtschaftswachstums führten im Jahresdurchschnitt zu fallenden Rohölpreisen. Der im Jahr 2019 immer wieder eskalierende Handelskonflikt zwischen China und den USA reduzierte das globale Wirtschaftswachstum und verringerte die globale Nachfrage nach Industrierohstoffen. Die Weltbank prognostizierte für 2019 ein Wirtschaftswachstum von 2,4\%, was dem schwächsten Wachstum seit der Finanzkrise entspricht. ${ }^{11}$ Das kürzlich unterzeichnete Teilabkommen zwischen den USA und China ließ die Preise für Industrieund Agrarrohstoffe wieder ansteigen und stärkt die Hoffnung auf eine Erholung der globalen Wirtschaft.

Claudia Wellenreuther wellenreuther@hwwi.org

10 Vgl. International Coffee Organization (ICO): Coffee Development Report 2019, Growing for prosperity, 2019, https://www.internationalcoffeecouncil.org/media/coffeeDevelopmentReport.pdf (3.2.2020).

11 Vgl. Weltbank, a.a. O. 